\title{
Article \\ Intelligent Prediction of Prestressed Steel Structure Construction Safety Based on BP Neural Network
}

\author{
Haoliang Zhu * and Yousong Wang
}

check for updates

Citation: Zhu, H.; Wang, Y. Intelligent Prediction of Prestressed Steel Structure Construction Safety Based on BP Neural Network. Appl. Sci. 2022, 12, 1442. https://doi.org/ 10.3390/app12031442

Academic Editors: Inhan Kim and Jung In Kim

Received: 29 December 2021

Accepted: 25 January 2022

Published: 28 January 2022

Publisher's Note: MDPI stays neutral with regard to jurisdictional claims in published maps and institutional affiliations.

Copyright: (C) 2022 by the authors. Licensee MDPI, Basel, Switzerland. This article is an open access article distributed under the terms and conditions of the Creative Commons Attribution (CC BY) license (https:// creativecommons.org/licenses/by/ $4.0 /)$.
School of Civil Engineering and Transportation, South China University of Technology, Guangzhou 510640, China; yswang@scut.edu.cn

* Correspondence: zhuhaoliang86@163.com

\begin{abstract}
In the construction process of a prestressed steel structure, it is a point of research interest to obtain the safety state of the structure according to the design parameters and working conditions of the structure. The intelligent prediction of structural construction safety provides the basis for safety control. This study proposes an intelligent prediction method of structural construction safety based on a back propagation (BP) neural network. Firstly, the correlation mechanism of structural construction safety performance parameters is established, which involves structural design parameters and mechanical parameters. According to the basic principle of a BP neural network, the relationship between design parameters and mechanical parameters is captured. The virtual model of a structure construction process is established based on digital twins (DTs). The DTs and BP neural network are combined to form a structural safety intelligent prediction framework and theoretical method, setting working conditions in a twin model to obtain mechanical parameters. Mechanical parameters are intelligently predicted by design parameters in neural networks. The safety performance of structure construction is evaluated according to mechanical parameters. Finally, the intelligent prediction method is applied to the construction process of string beam. Based on DTs and BP neural network, the intelligent analysis of structural construction safety is carried out. This provides a reliable basis for safety control. The feasibility of this research method is verified by comparing the predicted results of the theoretical method with the measured data on site.
\end{abstract}

Keywords: prestressed steel structure; BP neural network; digital twin; construction safety; intelligent prediction

\section{Introduction}

Prestressed steel structure has the advantages of a strong spanning capacity, beautiful shape, being light weight, and a short construction period, which is widely used in practical engineering [1]. The construction of a large-span spatial structure is also an important standard to measure national construction technology and its level. The mechanical properties of components directly determine the safety performance of the structure [2]. Large span spatial structure is mostly used in high importance buildings. Structure construction requires higher precision and safety. The research on its construction forming and safety control has also become a hot topic in the field of civil engineering. Due to the large volume in the construction process, the safety performance of the structure in the construction process is strictly required [3-5].

Luo et al. [6] systematically studied and applied construction control technology, the construction method, and construction mechanics analysis of cable tension. The bearing capacity analysis and experimental study were carried out on a cable pin shaft ear plate connection. Guo et al. [7] studied the influence of temperature action and cable length error on tension safety of a prestressed steel structure. Liu et al. [8] proposed an intelligent identification method of mechanical parameter sensitivity of a prestressed cable to analyze the safety performance of the structure. Arezki et al. [9] investigated the effect 
of temperature variation on the safety performance of cable truss structures and cables. Basta et al. [10] studied the quantitative evaluation of the decomposability of the cable-net structure based on building information modeling (BIM). Goh et al. [11] studied the simulation of a modular prestressed steel structure construction process based on lean production theory. Thai et al. [12] carried out the static and dynamic analysis of cable structure and studied the influence of an initial cable length error.

In the construction process of a large prestressed steel structure, due to the complex stress, high risk of aerial work, and large construction technology difficulty, construction safety is extremely important. The complex and changeable internal and external factors during construction will inevitably bring safety uncertainty to project implementation. This also affects the judgment of structural construction safety risks and the formulation of control decisions [13]. For the analysis of structural construction safety, it is necessary to integrate multi-source data to form an efficient and accurate safety control mechanism. The traditional analysis method does not consider using the virtual model to map the state of the real structure, and does not integrate multiple elements of time and space in the construction process. Therefore, real-time intelligent analysis of structural safety cannot be realized. In the construction process, how to predict the mechanical parameters of the structure through the design parameters of the structure so as to realize the intelligent analysis of the safety state is the focus of this study. Artificial intelligence has been applied in many disciplines and has formed a variety of intelligent algorithms. The algorithm can extract high-level features from the original data for perceptual decision-making, and improve the objectivity and accuracy of information evaluation [14]. Digital twins (DTs) is a key enabling technology to realize multi-dimensional and multi-scale information fusion, which has received extensive attention. Building a high-fidelity model based on the concept of DTs has become a point of interest in current research [15]. The application of intelligent algorithm and DTs concept in engineering practice can significantly improve the accuracy of structural safety performance prediction and control [16].

Wu et al. [17] established a radial basis function (RBF) artificial neural network (ANN) model to predict the 28-day compressive strength of concrete. Guided by the concept of DTs, Hou et al. [18] improved the performance of building health and safety through the internet of things and visualization technology. Lu et al. [19] proposed a DTs system architecture for the design of buildings and cities. The system supports the decision-making process in operation and maintenance management. Li et al. [20] used the improved particle swarm optimization (IPSO) to adjust the weights and thresholds of a back propagation (BP) neural network. The safety control and early warning model of damaged steel structure reconstruction construction is constructed. Han et al. [21] established a fracture identification model based on a deep convolution neural network. Love et al. [22] established a prediction model for quality and safety issues before construction based on the negative binomial regression model.

Aimed at the demand of intelligent prediction and control of prestressed steel structure construction safety, this study proposes an intelligent prediction method of prestressed steel structure construction safety based on a BP neural network. This research method combines the advantages of DTs and intelligent algorithms. According to the construction characteristics of the structure, the safety performance parameters are divided into structural design parameters and mechanical parameters. The correlation mechanism of two kinds of parameters is established. Based on the basic principle of a BP neural network, the construction safety performance parameters of the structure are integrated into the network. The intelligent prediction model of structural construction safety is formed. Combined with the characteristics of DTs, the simulation mapping of the construction process is carried out. The establishment of the twin model provides a basis for obtaining safety performance parameters. At the same time, the $\mathrm{BP}$ neural network is used for data analysis to intelligently predict the safety of the structure. Finally, the structural construction insecurity was judged in advance. The maintenance measures are formed in the twin model to guide the safety control of the construction site. The structure safety prediction mechanism driven by the 
BP neural network and DTs fusion is formed. Taking the string beam construction as the research object, the method is applied. The validity of the method is verified by comparing the experimental data and predicted data of the theoretical method.

\section{Methods}

\subsection{Fusion of Safety Performance Parameters and BP Neural Network}

Prestressed cable is introduced into a space prestressed steel structure. It is necessary to apply prestress to the structure to form geometric stiffness so as to withstand external load $[23,24]$. How to effectively use the abundant monitoring data and related safety risk information on the construction site to study the safety risk prediction and control of the whole process of prestressed steel structure construction becomes very important.

\subsubsection{Correlation of Structural Safety Performance Parameters}

In the construction process of large prestressed steel structure, due to the complex stress, high risk of aerial work, and large construction technology difficulty, construction safety is extremely important. Especially in the cable tension core link of a large span prestressed steel structure construction process, structural safety risk involves multiple factors. The complex and changeable internal and external factors during construction will inevitably bring safety uncertainty to the project implementation. This also affects the judgment of structural construction safety risks and the formulation of control decisions.

In the process of structural construction safety prediction, the corresponding relationship between design parameters and mechanical parameters is extracted according to the simulation results. With the help of a deep learning algorithm, association rules between data are mined. According to the characteristics of prestressed steel structure, the correlation mechanism between design parameters and mechanical parameters is formed in the construction stage of the structure, which is expressed as Equation (1):

$$
f\left(a_{1}, a_{2}, \cdots, a_{m}\right) \stackrel{R}{\Leftrightarrow} g\left(b_{1}, b_{2} \cdots, b_{n}\right) .
$$

In the Equation, $\left(a_{1}, a_{2}, \cdots, a_{m}\right)$ denotes the set of design parameters. $a_{1}, a_{2}, \cdots, a_{m}$ means the specific design parameters, such as the size of the member, the initial tension of the cable, the number of the structural pieces, etc., and $g\left(b_{1}, b_{2} \cdots, b_{n}\right)$ represents the aggregate of mechanical parameters. $b_{1}, b_{2} \cdots, b_{n}$ respectively equals to specific mechanical parameters, such as cable force, stress, and vertical displacement of structure. $\stackrel{R}{\Leftrightarrow}$ denotes the corresponding relationship between different design parameters and mechanical parameters according to technical standards and data association rules of structures. Driven by association rules, the intelligent prediction of structural construction safety can be realized. The mechanical parameters of the structure can be obtained by changing the design parameters, and the safety of the structure in the construction process can be judged.

\subsubsection{Basic Principle of BP Neural Network}

An artificial neural network has the advantages of self-adaptation, self-organization, strong robustness, and high efficiency, which can deal with linear programming, nonlinear programming, geometric programming, dynamic programming, and stochastic programming $[25,26]$. At present, the most widely used artificial neural network has more than 10 types, the most representative being the BP neural network. This algorithm has the ability to preliminarily analyze the original data and to repeatedly adjust the process parameters to achieve the adaptive processing consistent with the desired purpose. It is widely used in engineering, finance, chemical industry, energy, communication, and other fields, and has been continuously studied, optimized, and improved [27,28].

In practical application engineering, neural networks are often used in combination with some learning algorithms because different learning algorithms represent different thinking processes in human brain activities. A BP neural network is an intelligent data processing model used by a BP algorithm and neural network [29]. From the perspective of 
perceptron, a neural network can be divided into the input layer, hidden layer, and output layer [30]. The network topology in the neural network simulates the connection between human brain neurons. The activation function is a tool for analysis instead of human brain neurons, as shown in Figure 1.

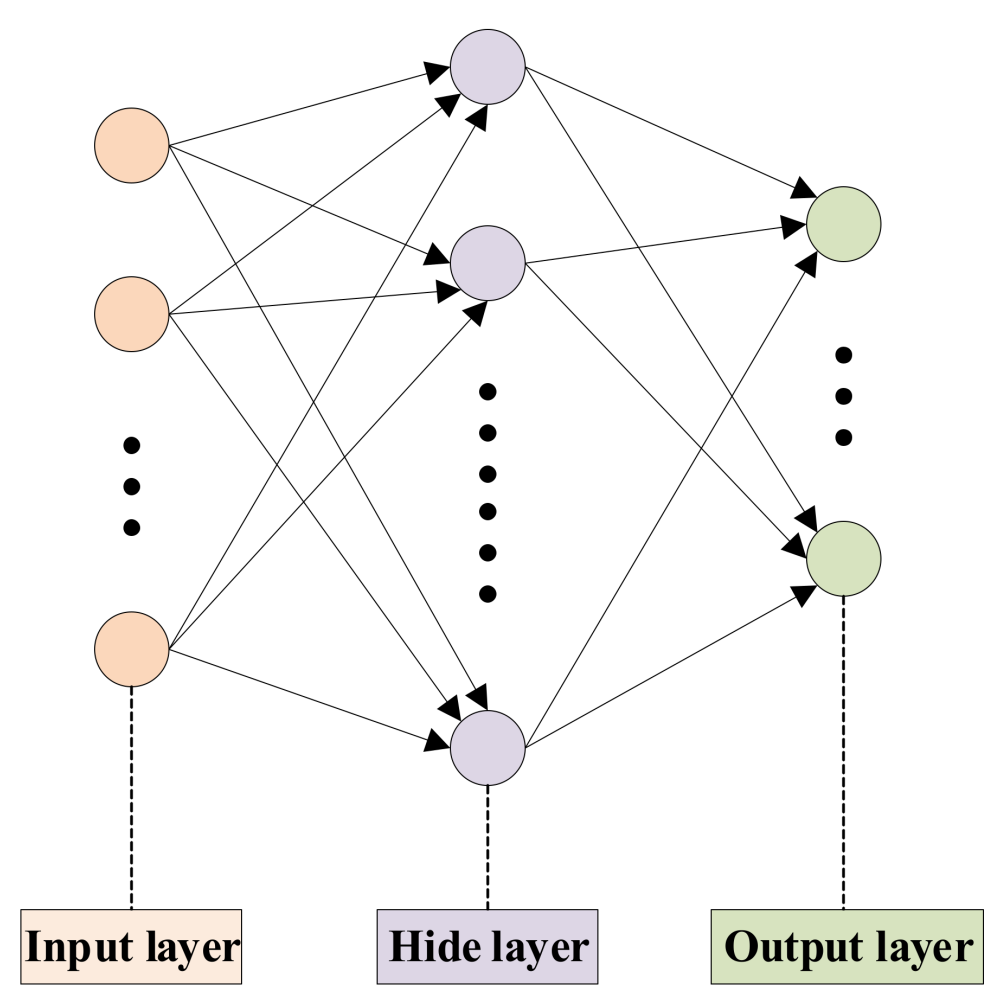

Figure 1. Neural network structure diagram.

From the perspective of function approximation, Equation (2) can be used to approximate any complex function. The nonlinear function represented by it can be realized by the BP network with a hidden layer without a bias value and linear transfer function of the output layer neurons:

$$
y_{k}=\sum_{j=1}^{N_{2}} \omega_{k j}^{2} f\left(\sum_{i=1}^{N_{1}} \omega_{j i}^{1} x_{i}+b_{j}\right) .
$$

In the Equation, $y_{k}$ is the $k$ th output. $\omega_{k j}^{2}$ is the weight of $j$-the neuron in the second layer (hidden layer), to $k$-the neuron in the output layer. $f(\cdot)$ is the transfer function of the hidden layer neurons. $\omega_{j i}^{1}$ is the weight of $i$-the neuron in the first layer (input layer), to $j$-the neuron in the hidden layer. $b_{j}$ is the bias value of $j$-the neuron in the hidden layer. $N_{1}$ is the number of neurons in the input layer. $N_{2}$ is the number of hidden neurons. As long as the number of hidden layer neurons is sufficient, the BP network with a hidden layer can approximate any complex nonlinear function with an arbitrary accuracy.

The nonlinear transfer function of BP neural network is usually taken as the Sigmoid function and expressed as Equation (3):

$$
f(x)=\frac{1}{1+e^{-\beta x}} .
$$

The BP neural network is a multi-input and multi-output network. The network realizes the mapping from $n$-dimensional input to $\mathrm{m}$-dimensional output. This mapping can be linear or nonlinear. This mainly depends on the type of transfer function used 
by neurons. The BP neural network realizes the pattern recognition function in the form of mapping.

\subsubsection{Fusion of Safety Parameters}

By analyzing the basic principle of the BP neural network, it provides a new idea for intelligent prediction of prestressed steel structure construction safety. In the process of structural safety prediction, the finite element software is used for simulation analysis [31]. Firstly, the design parameters of the structure are selected and the construction conditions of the structure are adjusted to obtain the corresponding mechanical parameters. A large number of samples are formed during finite element analysis. The sample contains the design parameters and mechanical parameters of the structure, which provides data support for the intelligent prediction model of structural construction safety based on $\mathrm{BP}$ neural network. According to the characteristics of prestressed steel structure, the aggregate of structural design parameters and mechanical parameters selected in this study is specifically expressed as Equations (4) and (5):

$$
\begin{gathered}
D P=\left(D_{u c b}, D_{s}, D_{c}, N_{s}, A_{c}, I T_{\mathcal{c}}\right) \\
M P=\left(D_{p}, S_{c}\right) .
\end{gathered}
$$

In the Equation, $D P$ represents the set of structural design parameters. $D_{u c b}$ equals to the size of the upper chord beam in the structure. $D_{s}$ means the size of the strut. $D_{c}$ is the diameter of the lower chord cable. $N_{S}$ represents the root number of struts in the structure. $A_{\mathcal{C}}$ denotes the radian of the lower chord cable to determine the length of the strut. $I T_{\mathcal{C}}$ represents the initial tension of the lower chord cable. $M P$ is the set of structural mechanical parameters, $D_{p}$ represents the maximum vertical displacement of the structure, and $S_{c}$ equals to the stress of the lower chord cable. The selection of design parameters provides the basis for building the simulation model, and the selection of mechanical parameters provides the basis for the feasibility of structural safety prediction.

In the construction safety prediction of prestressed steel structure, the initial parameters of the shape-finding model are determined first, including the number of nodes in the input layer, output layer, and hidden layer. In this process, based on the characteristics of structure construction, the type number of design parameters is selected as the number of input layer neurons, and the type number of mechanical parameters is selected as the number of output layer neurons. Before the prediction model is established, the simulation model is formed based on the selected design parameters to obtain the finite element results. The data from the finite element analysis are used as the training samples of the prediction model. By adjusting the network parameters, the error between the predicted value and finite element results is guaranteed to be within the threshold. Thus, the intelligent prediction model of structural construction safety is formed based on the BP neural network. In this model, the mechanical parameters of the structure can be accurately calculated and predicted by inputting design parameters. Based on the above content, the establishment of the intelligent prediction model of structural construction safety based on the BP neural network is shown in Figure 2.

\subsection{BP Neural Network and DTs Driven Structural Safety Prediction Mechanism}

To realize the intelligent prediction of construction safety performance, it is necessary to fuse the information of structure in multiple dimensions of time and space. DTs replicates the real physical entity through visual virtual space modeling, and simulates the dynamic behavior of the entity in the real environment by the virtual model [32]. The integration of BP neural network and DTs can realize the intelligent prediction and closed-loop control of structural construction safety. 


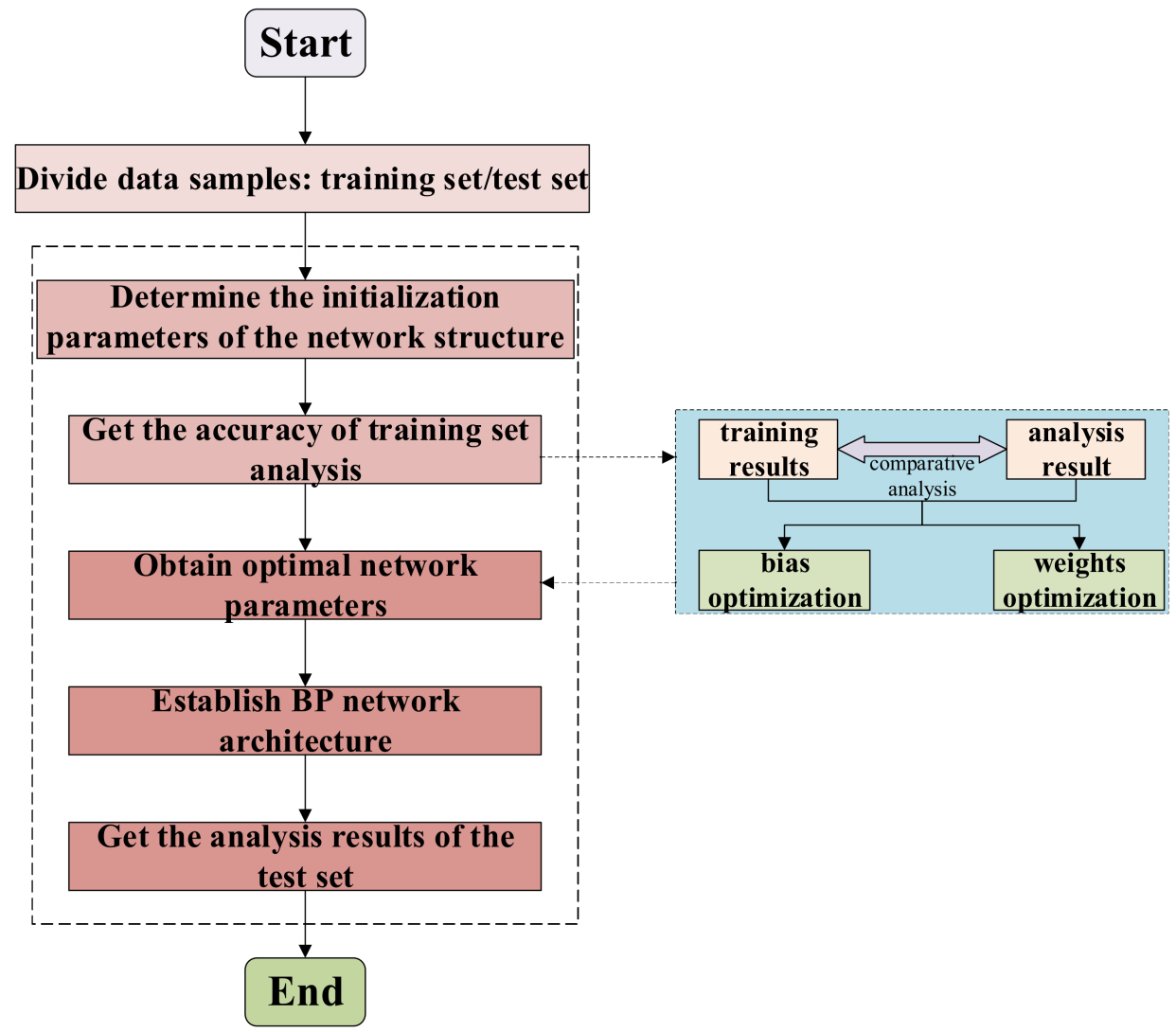

Figure 2. Construction safety intelligent prediction model establishment process.

\subsubsection{Fusion Mechanism of BP Neural Network and DTs}

Driven by DTs, the formation of multi-dimensional virtual model of the structural construction process is the basis of intelligent evaluation of structural safety [33]. The twin data are formed by the interactive feedback of the measured data in the physical space and the simulation data in the virtual space. Driven by the twin data, the dynamic perception and real-time control of the physical space can be realized. In the DTs modeling framework for construction safety prediction of prestressed steel structures, the construction of twinning model is divided into four levels of "geometry-physics-behavior-rule". According to the actual construction process of the construction site, each dimension model is correlated and integrated to realize the deep, multi-angle, and comprehensive simulation of the construction site.

The first step of virtual model establishment is modeling basic information of appearance, size, and type of components at the geometric level. The geometric model is mainly established by BIM modeling software, such as Revit [34]. By establishing a high-fidelity geometric model, the geometric characteristics of the construction process can be truly mapped. Simulation mapping provides strong support for the subsequent analysis of the physical model. During geometric modeling, a list of structural component properties is formed. This provides design parameter support for structural safety prediction. At the same time, in the BIM model, its editability can be used to modify the design parameters to directly obtain the structure shape. The geometric model based on BIM provides design parameter support for the establishment of a physical model. At the physical level, the material parameters of construction components are mainly simulated in the physical model by finite element analysis software, such as Midas. In this process, with the collected data of the sensing equipment, the geometric model and connection parameters of the components in the model are modified. The calculation of structural mechanical properties during construction is realized by the physical model in the end. The geometric model and physical model are established to describe the construction site and provide model 
support for the safety assessment. At the behavior level, the finite element model can set the working conditions to match the actual construction. The mechanical properties of components and changes of the parameters of the material itself under the action of working conditions are analyzed. The material parameters and mechanical properties' parameters extracted in this way can be directly used for the assessment of construction safety. At the same time, based on the establishment of BIM and mechanical analysis model, structural design parameters and mechanical parameters can be obtained. This provides data support for structural safety prediction based on a BP neural network. At the rule level, according to the standard specification, the mechanical properties' parameters of the components in the construction process should be quantitatively limited. The rule model is the reference standard for quality control, risk prediction, and decision optimization.

Based on the twin model of the construction process structure, the design parameters and mechanical parameters of the structure can be extracted in real time. By establishing the correlation mechanism of safety performance parameters, the data is input into the BP neural network. Driven by the BP neural network, a high robustness structural construction safety prediction model is established. According to the mechanical parameters output from the model, the safety performance of the structure is evaluated. For unsafe events, maintenance measures are formulated. Finally, the measures are input into the twin model to analyze the feasibility of safety maintenance. Thus, the intelligent prediction and closedloop control of structural construction safety are formed. The fusion mechanism of the BP neural network and DTs is shown in Figure 3.

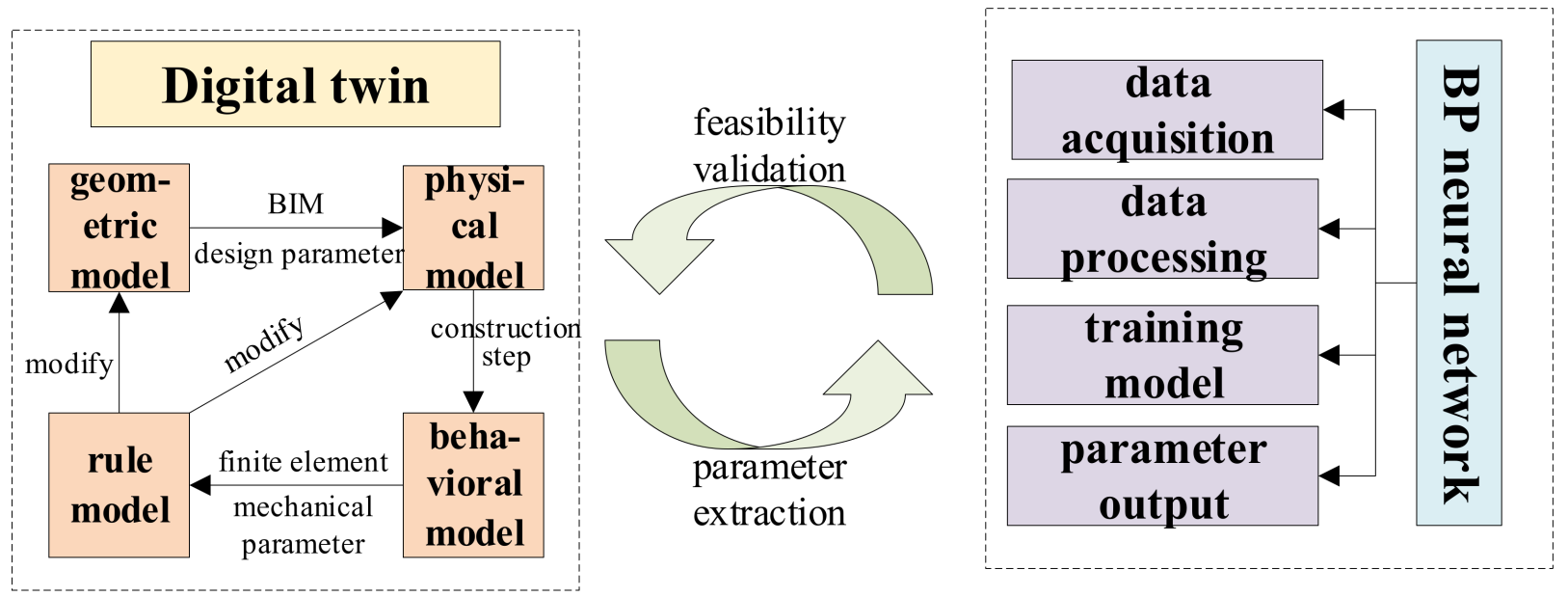

Figure 3. Fusion mechanism of back propagation neural network and digital twins.

\subsubsection{Structure Safety Intelligent Prediction Framework}

By establishing a high-fidelity virtual model, the structural mechanical performance of the actual construction process can be simulated in the virtual space. According to the working conditions in the actual construction process, the simulation is carried out in the virtual model to obtain the mechanical property information of the structure that is highly consistent with the actual structure. Based on the obtained safety performance parameters, a prediction model with high robustness is established by combining the BP neural network. By inputting the design parameters of the structure, the mechanical parameters of the structure are predicted intelligently and the safety performance of the structure is evaluated. For unsafe events, this method assists in formulating reliable maintenance measures to guide structural construction. The structure safety intelligent prediction framework driven by the fusion of BP neural network and DTs is shown in Figure 4 . 


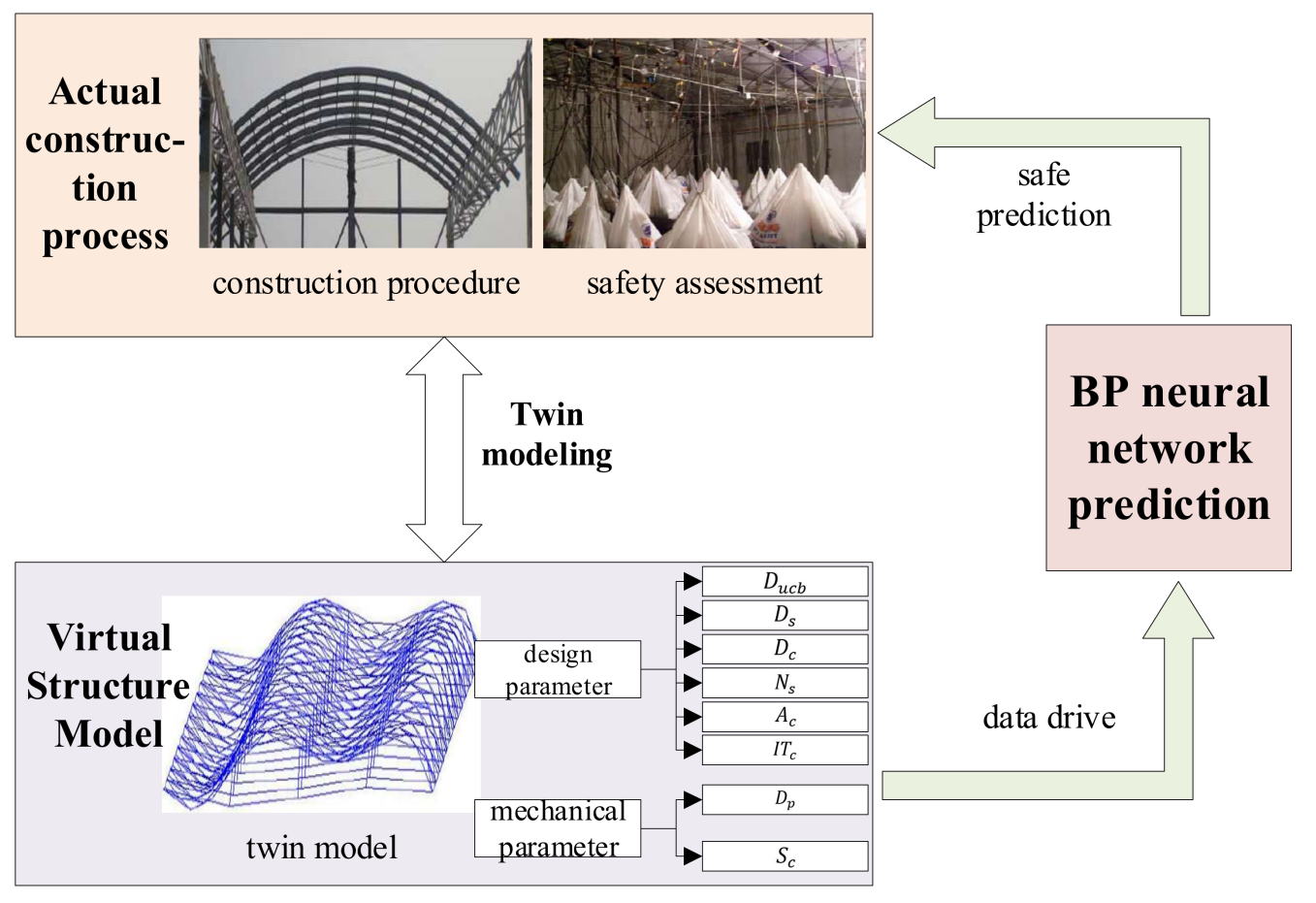

Figure 4. The framework structure safety intelligent prediction.

\section{Results}

The data of DTs modeling is applied to a BP neural network to form an intelligent prediction method for structural construction safety. This study applies the theoretical method to the construction process of beam string. The correlation mechanism between design parameters and mechanical parameters is established. Driven by this research method, the intelligent prediction of structural safety performance is realized. The effectiveness of this research method is verified by comparing with field data collection.

\subsection{Test Structure Model}

Taking the chord beam roof of a convention and exhibition center as the research object, one of the plane string structure is selected as the calculation model. One of the design parameters is taken in this study, and the model construction is shown in Figure 5.

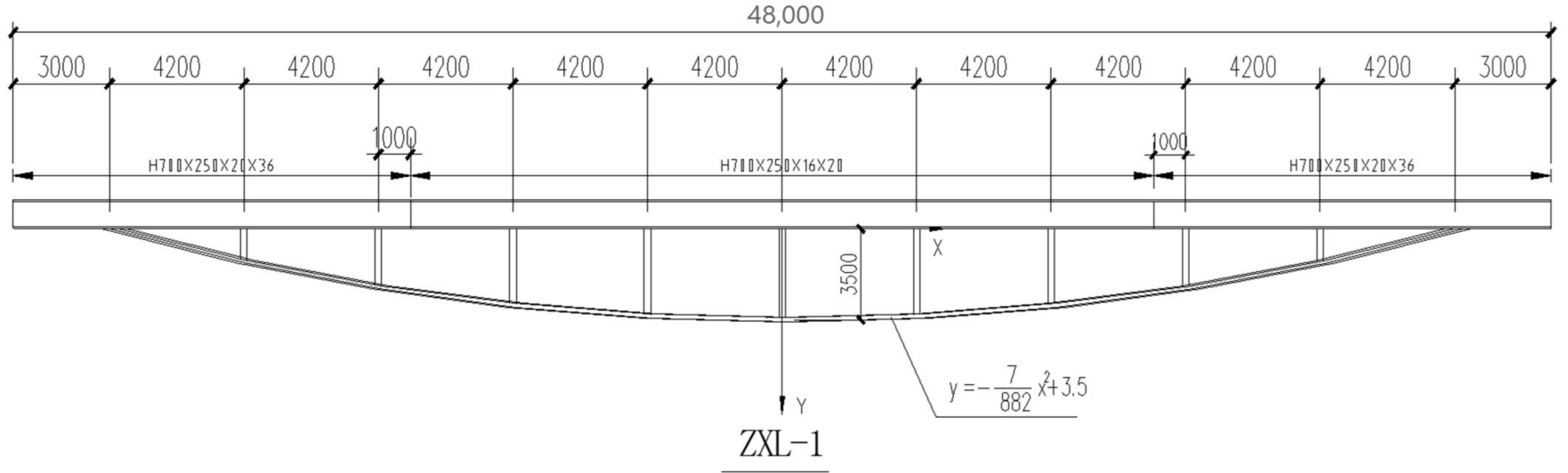

Figure 5. Structural model.

In the process of structural construction safety prediction, the size of the above chord beam, the size of the strut, the diameter of the lower chord cable, the number of the struts, 
the arc of the lower chord cable, and the initial tension of the lower chord cable are taken as the structural design parameters. The arc of the lower chord cable is used to reflect the length of each strut. In this test structure, the tangent value $(\tan \alpha)$ of the radian is expressed by Equation (6):

$$
\tan \alpha=\frac{h_{s}}{l_{c}} .
$$

In the Equation, $h_{s}$ represents the length of mid-span brace. $l_{c}$ means the distance from the starting point of the lower chord cable to the midspan.

According to the design parameters, the mechanical parameters of the structure can be generated by setting the construction conditions in the finite element software. The main mechanical parameters analyzed in this study are the vertical displacement and the stress of the lower chord cable. The working condition considered in the construction process is $0.9^{*}$ constant load $+1.5^{*}$ wind load. In the twin model, the mechanical parameters of the structure are shown in Figure 6.

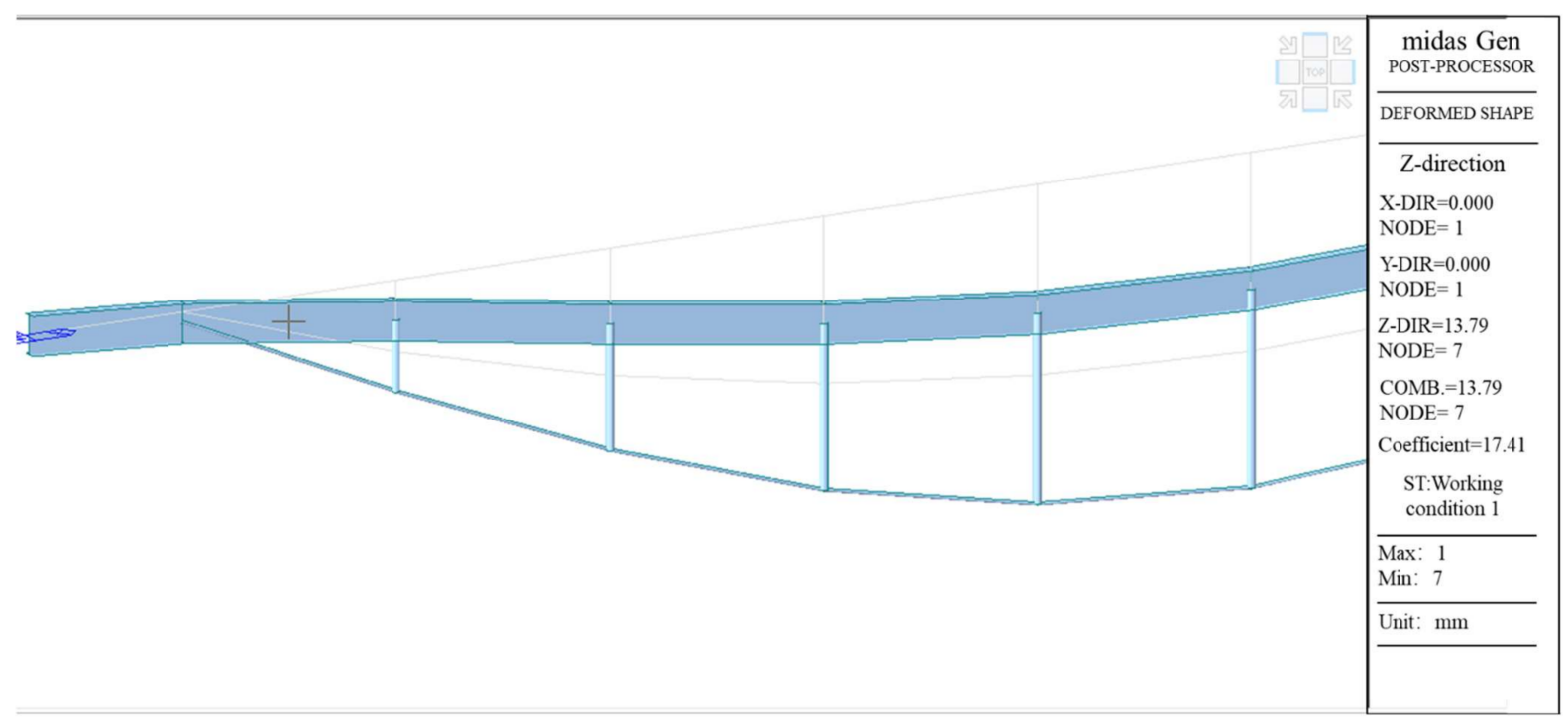

(a)

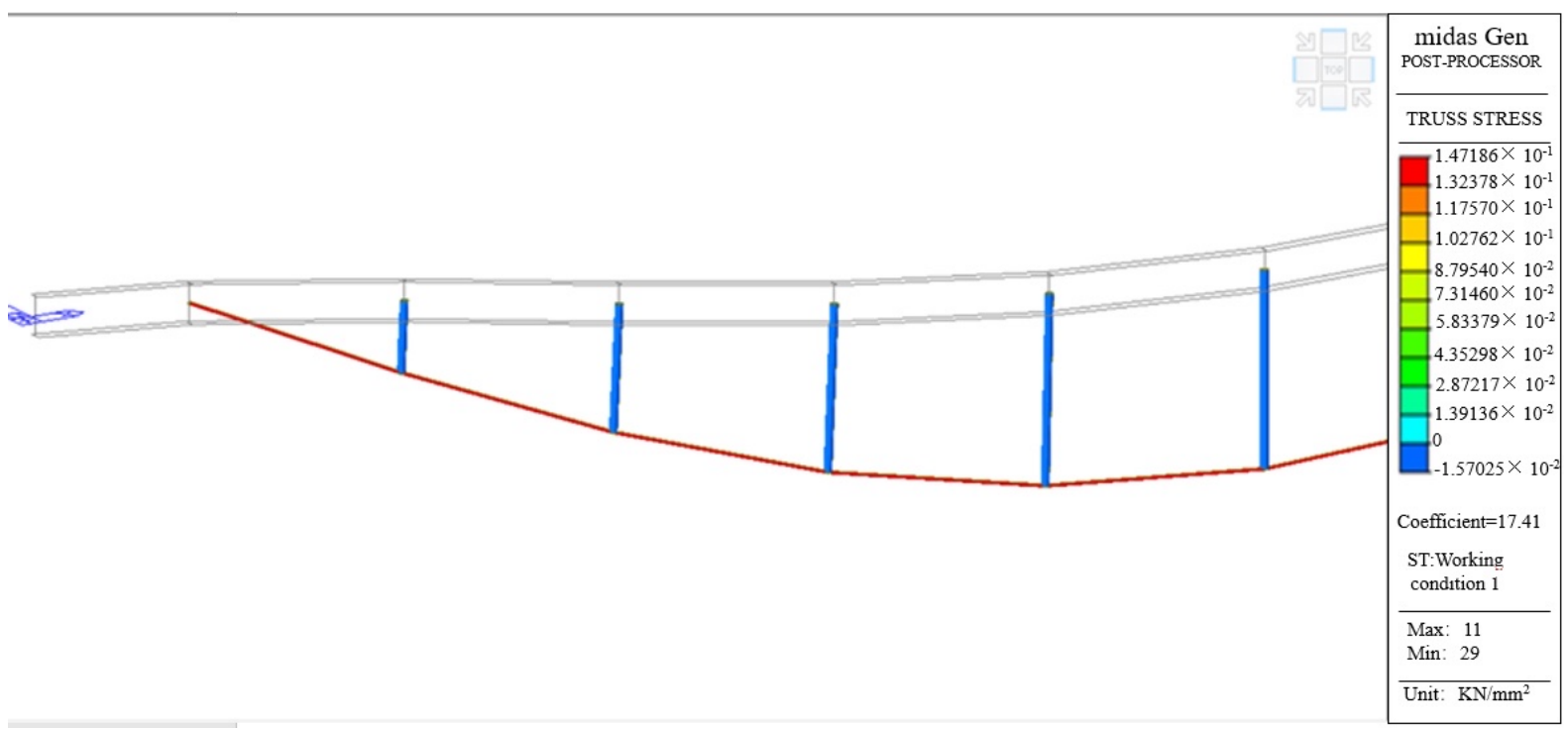

(b)

Figure 6. Mechanical parameters of the structure. (a) Perpendicular displacement. (b) Stress. 


\subsection{Establishment of Prediction Model}

According to the analysis of the basic principle of the BP neural network in Section 2.1.2, an intelligent prediction model for prestressed steel structure construction safety is established. The design parameters and mechanical parameters are used as the input layer and output layer of the neural network, respectively. The data obtained by the finite element under the self-weight condition of the structure are used as the samples of the training prediction model. In this study, a hidden layer was established, in which the number of neurons in the hidden layer was determined by Equation (7) [35]:

$$
l=\sqrt{n+m}+a .
$$

Among them, $l$ is the number of hidden neurons. $n$ is the number of neurons in the input layer, namely the number of types of design parameters. $m$ is the number of neurons in the output layer, namely the number of types of mechanical parameters. $a$ is the constant between $[1,10]$. The number of iterations of the BP neural network is 6000 , and the learning rate is 0.035 . The model selects $m s e$ as the performance function of the evaluation model, and ends the iteration when $m s e \leq 6.5 \times 10^{-4}$. In the construction process of this structure, in order to accurately predict the mechanical parameters, two prediction models of the vertical displacement and stress of the lower chord cable corresponding to the design parameters are established, respectively. According to engineering experience, the values of six design parameters are shown in Table 1.

Table 1. Values of design parameters.

\begin{tabular}{cc}
\hline Parameter Type & Taking Values \\
\hline Size of upper chord beam $(\mathrm{mm})$ & $\mathrm{H} 700 \times 250 \times 20 \times 36, \mathrm{H} 700 \times 250 \times 16 \times 20$ \\
Size of strut $(\mathrm{mm})$ & $\varnothing 150 * 8, \varnothing 160 * 8, \varnothing 170 * 8$ \\
Diameter of lower chord cable $(\mathrm{mm})$ & $50,55,60,65,70$ \\
Number of the strut & $7,9,11$ \\
Arc of lower chord cable $\left(^{\circ}\right)$ & $8,9,10$ \\
Initial tension of the lower chord cable $(\mathrm{KN})$ & $600,700,800,900,1000$ \\
\hline
\end{tabular}

According to the value of design parameters, 150 samples are formed under the condition of self-weight. In the process of training the prediction model, the influence of the number of neurons in different hidden layers on the number of iterations is discussed. When $m s e \leq 6.5 \times 10^{-4}$, the model stops iteration. In this study, the effects of six types of design parameters on the vertical displacement and stress of the lower chord cable are studied respectively. Therefore, in each neural network, $n=6, m=1$. According to Equation (7) in each neural network, $l$ takes 10 integers between 4 and 13. The effect of the number of neurons in different hidden layers on the number of iterations is shown in Figure 7.

In the process of neural network training, the less the number of iterations, the higher the efficiency of calculation. At the same time, the corresponding prediction accuracy is also higher. According to Figure 7, for the prediction of vertical displacement and lower chord cable stress, the number of hidden layer nodes respectively takes 11 and 6 corresponding to the least number of iterations, and the accuracy of the model is the highest. Thus, the number of nodes corresponding to the neural network for different mechanical parameters in the prediction of structural safety performance is determined. The comparison between the predicted values $(\mathrm{PV})$ of mechanical parameters output by the final prediction model and the actual values (AV) of mechanical parameters at the construction site are shown in Figures 8 and 9. 


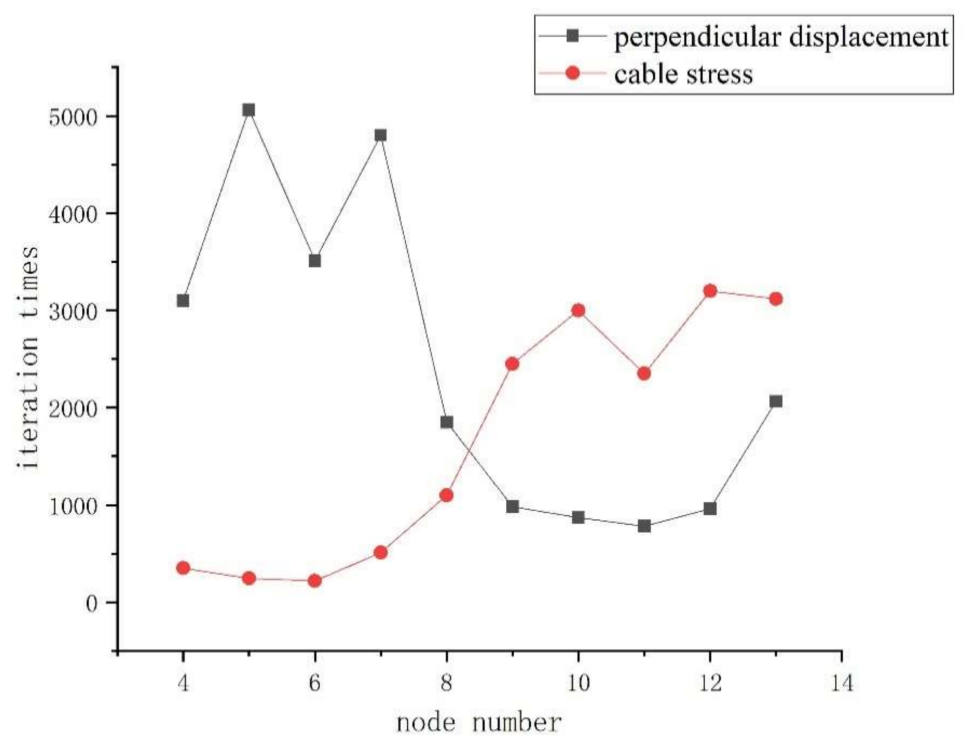

Figure 7. Effect of different number of hidden layer neurons on the iteration number.

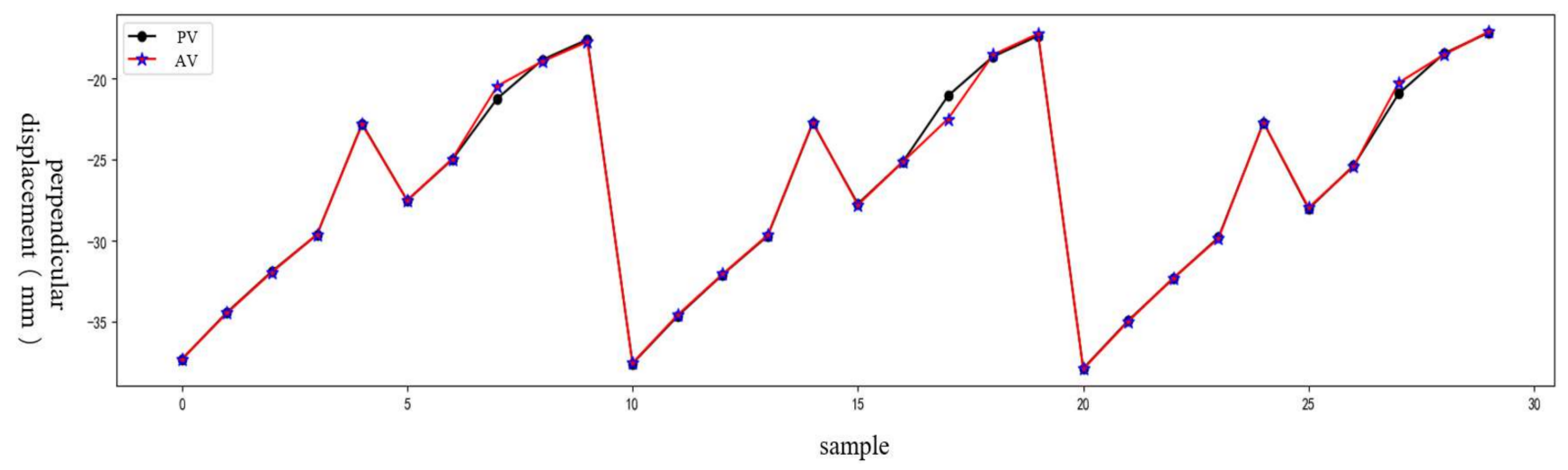

Figure 8. Comparison of the vertical displacement output from the prediction model with the actual vertical displacement (part of the samples).

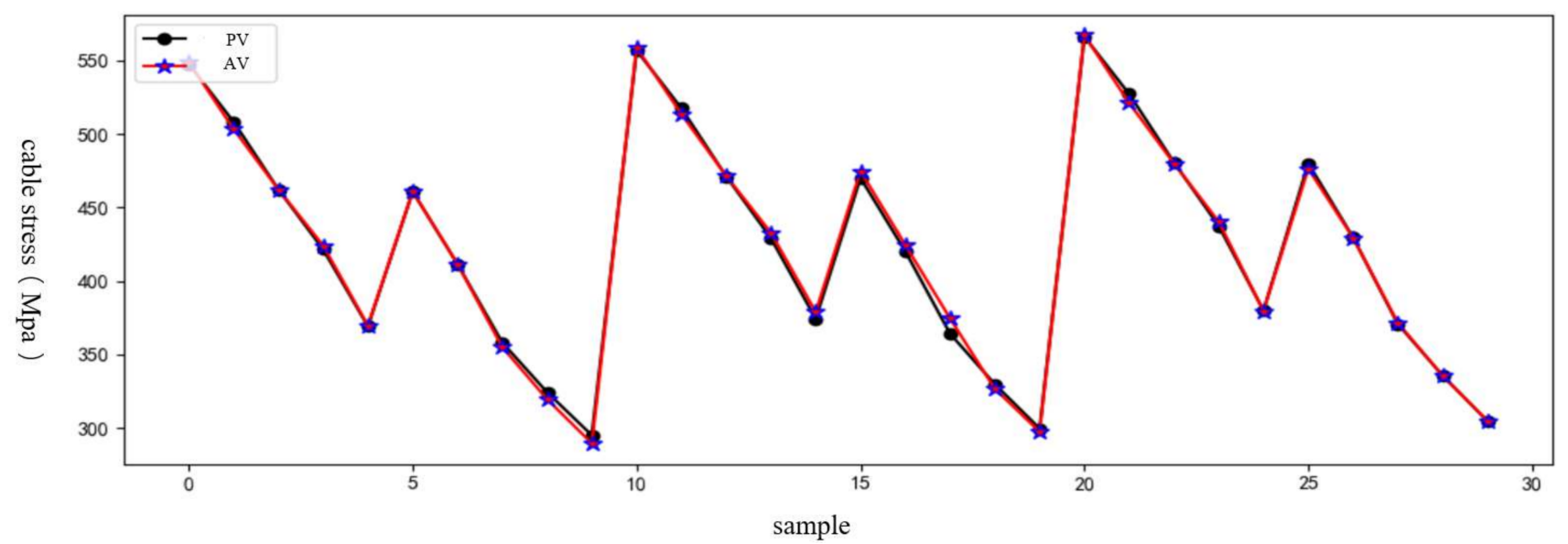

Figure 9. Comparison of the cable stress output from the prediction model with the actual cable stress (part of the samples). 


\subsection{Changes of Safety Performance under Construction Conditions}

Based on the safety prediction model established by 3.2, the safety performance of the selected construction conditions is predicted. In the case of self-weight, the number of hidden layer nodes in the neural network model for vertical displacement and cable stress is trained. The construction condition considered in the construction process of the structure is $0.9^{*}$ constant load $+1.5^{*}$ wind load. In the research process, two aspects of mechanical parameters data are formed. On the one hand, the design parameters of the structure are modified by the twin model, and the mechanical parameters of the structure are obtained by arranging the construction conditions. The resulting data is regarded as the data set of the neural network. In the neural network, the design parameters of the twin model are used as the input layer and the mechanical parameters are used as the output layer. Thus, the reliable prediction model is obtained by training, and finally the mechanical parameters of the test set are generated to predict the safety of the structure. On the other hand, the design parameters of the test set are used to form a realistic construction structure, and the construction conditions are set at the site to obtain the corresponding mechanical parameters. In order to verify the validity of the model, the comparison between the predicted values (PV) of mechanical parameters output by the final prediction model and the actual values $(\mathrm{AV})$ of mechanical parameters at the construction site are shown in Figures 10 and 11.

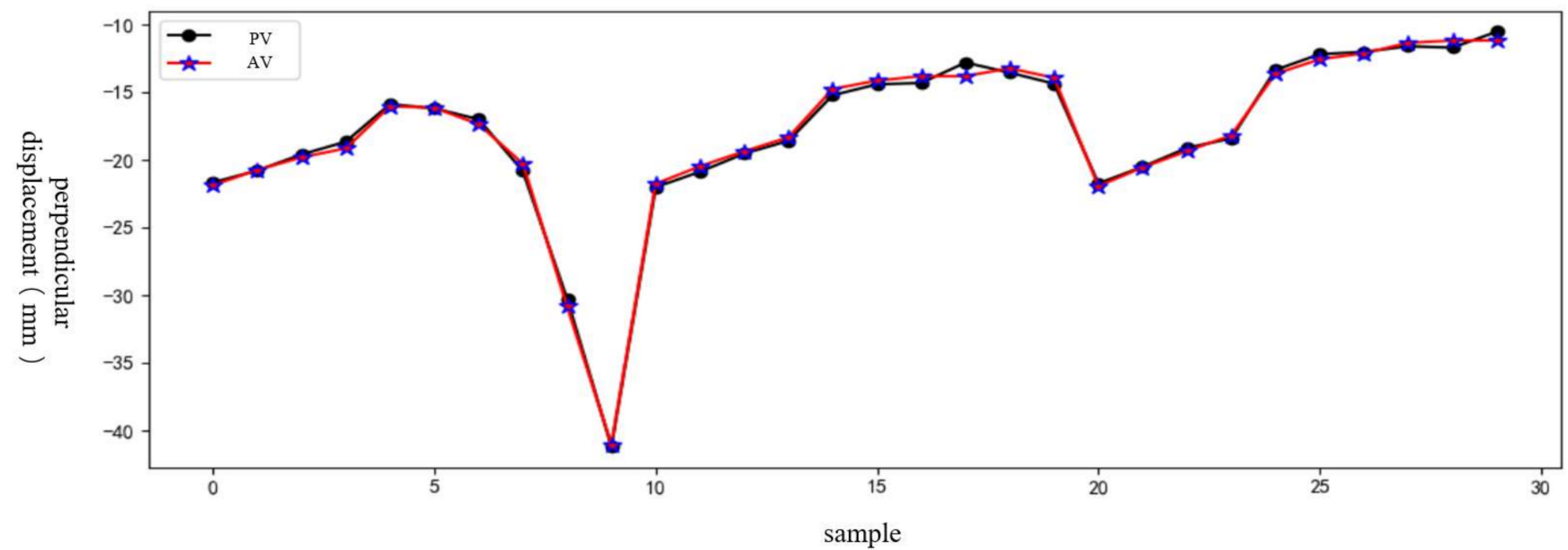

Figure 10. Comparison of the model output vertical displacement with the measured value (part of the samples).

Through the integration of DTs and BP neural network, the intelligent prediction mechanism of structural construction safety is formed. The design parameters and mechanical parameters of construction process structure are extracted by the digital twin model. Driven by the BP neural network, a high robustness safety performance prediction model is established. Thus, the structural design parameters can be directly input to generate the mechanical parameters of the structure, and the intelligent prediction of structural safety performance can be realized. The safety performance of the structure is judged according to the limit value of mechanical parameters. In the construction process, the limit values of the mechanical parameters of the structure are shown in Table 2 . This study provides a basis for structural safety control. During the test, there is no case of mechanical parameters exceeding the limit, so it is not necessary to maintain the structure.

By comparing the predicted and measured values of structural mechanical parameters, the feasibility of predicting structural safety performance the by twin model and BP neural network is verified. The construction conditions are set in the twin model to extract the training set and test set data required by the neural network. The structural safety prediction model is formed by BP neural network, and the mechanical parameters characterizing structural safety are obtained, so as to intelligently analyze the change of safety performance 
under construction conditions. This research method realizes the prediction of structural construction safety and provides a basis for structural safety control.

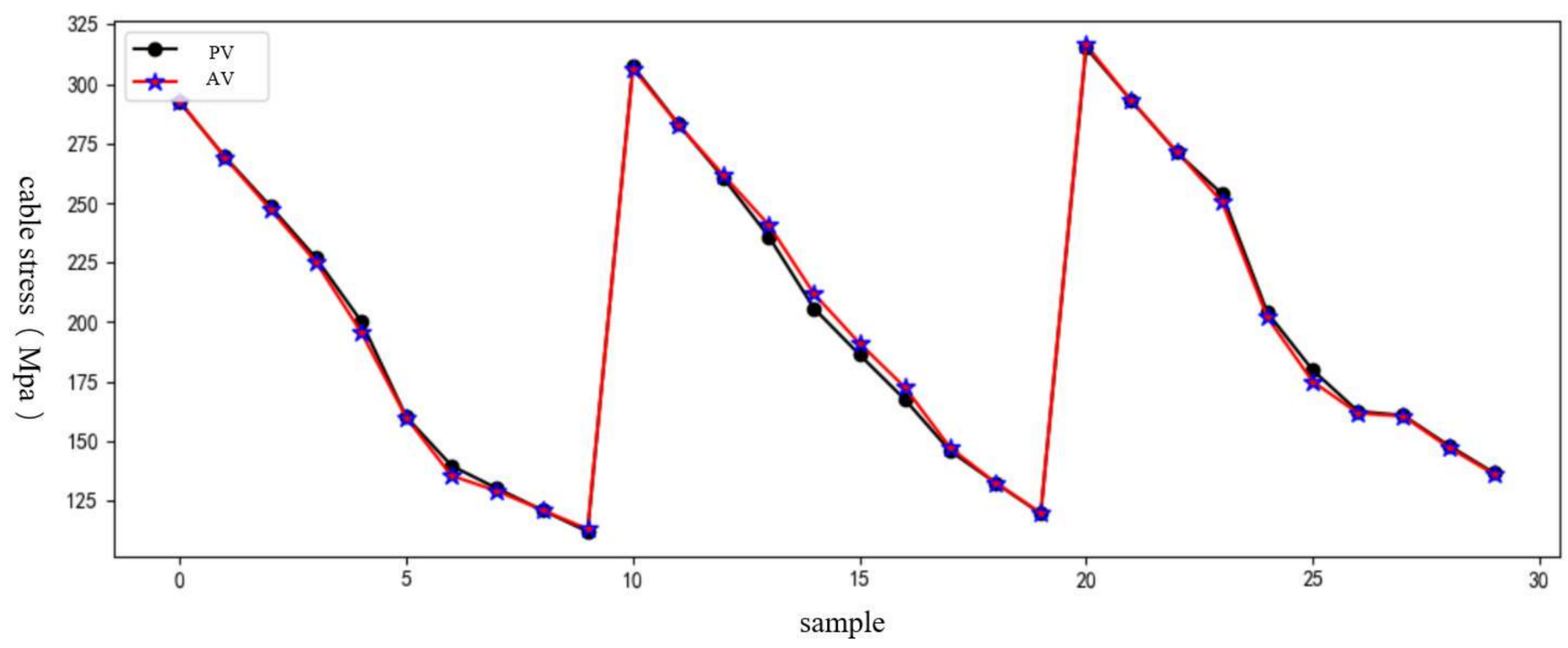

Figure 11. Comparison between the model output cable stress and measured value (part of the samples).

Table 2. Limits of mechanical parameters of structures.

\begin{tabular}{cc}
\hline Mechanical Parameter & Specific Limits \\
\hline Perpendicular displacement & less than $1 / 250$ of structural span \\
Cable stress & less than $1 / 2.5$ of allowable stress \\
\hline
\end{tabular}

\section{Discussion and Conclusions}

In the process of structural construction, it is a research hotspot to intelligently predict the mechanical parameters of the structure and then predict the safety performance of the structure according to the design parameters. This study proposes an intelligent prediction method of structural construction safety based on the BP neural network. Based on the exploration of the theoretical method, it is applied in the construction process of string beam. The feasibility of this research method is verified by comparing the predicted and measured values of mechanical parameters. In the research process of structural construction safety intelligent analysis method, the following main findings were obtained.

(1) Based on the construction characteristics of prestressed steel structure, the correlation mechanism of structural safety performance parameters is established. Driven by the BP neural network, the structural design parameters and mechanical parameters are integrated.

(2) In the process of structural safety prediction, the virtual-real interaction is carried out by DTs. The simulation data are extracted in the twin model. The fusion mechanism of DTs and BP neural network is formed for the intelligent prediction of structural construction safety. Supported by each other, a high robustness structural construction safety performance prediction model is established.

The construction conditions are set in the digital twin model, and the design parameters and mechanical parameters of the structure are extracted to provide data samples for the neural network. The mechanical parameters of the intelligent prediction structure formed by the BP neural network are used to analyze its safety state. In the construction process, a structural safety assessment is carried out according to the changes of mechanical parameters of different structural forms. This research method improves the intelligent degree of structural construction safety prediction. At the same time, the integration of mechanical parameters and specification constraints provides a reference for the control of 
the construction safety of real structures. Combining mechanical parameters with design parameters to find the key factors that affect the structural safety performance is the next research focus. Therefore, based on the experience and lessons of this study, future research is needed to improve the intelligence level of the construction process by considering more realistic conditions.

Author Contributions: Conceptualization, H.Z.; methodology, H.Z.; software, H.Z.; validation, H.Z. and Y.W.; writing—original draft preparation, H.Z.; writing—review and editing, H.Z.; project administration, H.Z.; funding acquisition, H.Z. All authors have read and agreed to the published version of the manuscript.

Funding: This research received no external funding.

Institutional Review Board Statement: Not applicable.

Informed Consent Statement: Not applicable.

Data Availability Statement: The data presented in this study are available on request from the corresponding author. The data are not publicly available due to the confidentiality.

Acknowledgments: The authors would like to thank South China University of Technology, Guangzhou, China, for their support throughout the research project.

Conflicts of Interest: The authors declare no conflict of interest. The authors declare no conflict of interest. The funders had no role in the study's design; in the collection, analyses or interpretation of data; in the writing of the manuscript or in the decision to publish the results.

\section{References}

1. Zhang, A.L.; Sun, C.; Jiang, Z.Q. Calculation method of prestress distribution for levy cable dome with double struts considering self-weight. Eng. Mech. 2017, 34, 211-218. (In Chinese)

2. Krishnan, S. Structural design and behavior of prestressed cable domes. Eng. Struct. 2020, 209, 110294. [CrossRef]

3. Shekastehband, B.; Abedi, K.; Dianat, N.; Chenaghlou, M. Experimental and numerical studies on the collapse behavior of tensegrity systems considering cable rupture and strut collapse with snap-through. Int. J. Non-Linear Mech. 2012, 47, 751-768. [CrossRef]

4. Ge, J.Q.; Liu, B.N.; Wang, S.; Zhang, G.J.; Zhang, M.S.; Huang, J.Y.; Liu, X.G. Study on design of prestressed tensegrity cable structures. J. Build. Struct. 2019, 40, 73-80. (In Chinese)

5. Chen, Z.H.; Ma, Q.; Yan, X.Y.; Lou, S.Y.; Chen, R.H.; Si, B. Research on Influence of Construction Error and Controlling Techniques of Compound Cable Dome. J. Hunan Univ. (Nat. Sci.) 2018, 45, 47-56. (In Chinese)

6. Wang, Z.H.; Luo, B.; Zhang, M.Q.; Zhang, Y.Q.; Zhang, C.H. Experimental study and finite element analysis on bearing capacity of pin-connected plate joints. J. Build. Struct. 2020, 41, 150-159. (In Chinese)

7. Guo, Y.L.; Zhang, X.Q. Influences of temperature changes and cable length errors on tension structures using un-adjustable cable length design. China Civ. Eng. J. 2017, 50, 11-22, 61. (In Chinese)

8. Liu, Z.; Shi, G.; Jiang, A.; Li, W. Intelligent Discrimination Method Based on Digital Twins for Analyzing Sensitivity of Mechanical Parameters of Prestressed Cables. Appl. Sci. 2021, 11, 1485. [CrossRef]

9. Arezki, S.; Kamel, L.; Amar, K. Effects of temperature changes on the behavior of a cable truss system. J. Constr. Steel Res. 2017, $129,111-118$.

10. Basta, A.; Serror, M.H.; Marzouk, M. A BIM-based framework for quantitative assessment of steel structure deconstructability. Autom. Constr. 2020, 111, 103064. [CrossRef]

11. Goh, M.; Goh, Y.M. Lean production theory-based simulation of modular construction processes. Autom. Constr. 2019, 101, 227-244. [CrossRef]

12. Thai, H.-T.; Kim, S.-E. Nonlinear static and dynamic analysis of cable structures. Finite Elements Anal. Des. 2011, 47, 237-246. [CrossRef]

13. Harirchian, E.; Kumari, V.; Jadhav, K.; Raj Das, R.; Rasulzade, S.; Lahmer, T. A Machine Learning Framework for Assessing Seismic Hazard Safety of Reinforced Concrete Buildings. Appl. Sci. 2020, 10, 7153. [CrossRef]

14. Le Cun, Y.; Bengio, Y.; Hinton, G. Deep learning. Nature 2015, 521, 436-444. [CrossRef]

15. Tao, F.; Zhang, H.; Qi, Q.L.; Xu, J.; Sun, Z.; Hu, T.L.; Liu, X.J.; Liu, T.Y.; Guan, J.T.; Chen, C.Y.; et al. Theory of digital twin modeling and its application. Comput. Integr. Manuf. Syst. 2021, 27, 1-15.

16. Liu, Z.; Shi, G.; Zhang, A.; Huang, C. Intelligent Tensioning Method for Prestressed Cables Based on Digital Twins and Artificial Intelligence. Sensors 2020, 20, 7006. [CrossRef]

17. Wu, N.-J. Predicting the Compressive Strength of Concrete Using an RBF-ANN Model. Appl. Sci. 2021, 11, 6382. [CrossRef] 
18. Hou, L.; Wu, S.; Zhang, G.; Tan, Y.; Wang, X. Literature Review of Digital Twins Applications in Construction Workforce Safety. Appl. Sci. 2020, 11, 339. [CrossRef]

19. Lu, Q.; Parlikad, A.K.; Woodall, P.; Xie, X. Developing a dynamic digital twin at building and city levels: A case study of the West Cambridge campus. J. Manag. Eng. 2019, 36, 1-19.

20. Li, H.M.; Duan, P.S.; Meng, H.; Guo, H.D. Study on safety early-warning assessment of damaged steel structure reconstruction based on IPSO-BP. J. Saf. Sci. Technol. 2019, 15, 174-180. (In Chinese)

21. Han, X.J.; Zhao, Z.C. Structural surface crack detection method based on computer vision technology. J. Build. Struct. 2018, 39, 418-427. (In Chinese)

22. Love, P.E.D.; Toe, P. Statistical Analysis of Injury and Nonconformance Frequencies in Construction: Negative Binomial Regression Model. J. Constr. Eng. Manag. 2017, 143, 05017011. [CrossRef]

23. Zhang, Z.H.; Liu, L.; Zhi, X.D.; Fan, F. Study on stability of cable supported single-layer two-way grid cylindrical shell. J. Build. Struct. 2021, 42, 39-47. (In Chinese)

24. Cao, T.; D'Acunto, P.; Castellón, J.J.; Tellini, A.; Schwartz, J.; Zhang, H. Design of prestressed gridshells as smooth poly-hypar surface structures. Structures 2021, 30, 973-984. [CrossRef]

25. Liu, Z.; Jiang, A.; Shao, W.; Zhang, A.; Du, X. Artificial-Neural-Network-Based Mechanical Simulation Prediction Method for Wheel-Spoke Cable Truss Construction. Int. J. Steel Struct. 2021, 21, 1032-1052. [CrossRef]

26. Afram, A.; Janabi-Sharifi, F.; Fung, A.S.; Raahemifar, K. Artificial neural network (ANN) based model predictive control (MPC) and optimization of HVAC systems: A state of the art review and case study of a residential HVAC system. Energy Build. 2017, 141, 96-113. [CrossRef]

27. Chen, S.Y.; Han, J.P.; Yu, X.H.; Lv, D.G. Seismic Fragility Surface Analysis of Rc Frame Structures Based on Bp Neural Networks: Accounting for the Effects of Ground Motion Intensity and Duration. Eng. Mech. 2021, 38, 107-117. (In Chinese)

28. Ji, X.; Yang, B.; Tang, Q. Acoustic Seabed Classification Based on Multibeam Echosounder Backscatter Data Using the PSO-BPAdaBoost Algorithm: A Case Study from Jiaozhou Bay, China. IEEE J. Ocean. Eng. 2020, 46, 509-519. [CrossRef]

29. Lachhwani, K. Application of Neural Network Models for Mathematical Programming Problems: A State of Art Review. Arch. Comput. Methods Eng. 2019, 27, 171-182. [CrossRef]

30. Parisi, G.I.; Kemker, R.; Part, J.L.; Kanan, C.; Wermter, S. Continual lifelong learning with neural networks: A review. Neural Netw. 2019, 113, 54-71. [CrossRef]

31. Li, N.; Liao, J.; Lu, J.Y.; Zong, W. Configuration generation and mechanical properties analysis of circular-wave reticulated shells. J. Build. Struct. 2016, 37, 121-126. (In Chinese)

32. Angjeliu, G.; Coronelli, D.; Cardani, G. Development of the simulation model for Digital Twin applications in historical masonry buildings: The integration between numerical and experimental reality. Comput. Struct. 2020, 238, 106282. [CrossRef]

33. Liu, Z.; Shi, G.; Jiao, Z.; Zhao, L. Intelligent Safety Assessment of Prestressed Steel Structures Based on Digital Twins. Symmetry 2021, 13, 1927. [CrossRef]

34. Ma, Z.L.; Teng, M.K.; Ren, Y. Method of extracting static data for building energy consumption monitoring from BIM. J. Harbin Inst. Technol. 2019, 51, 187-193.

35. Guo, X.; Ma, S.Y.; Guo, Y.F. Blast peak pressure prediction for surrounding rock medium based on BP neural network method. J. Vib. Shock 2019, 38, 199-206. 